Article

\title{
Investigating Quantum Coherence by Negative Excursions of the Wigner Quasi-Distribution
}

\author{
Mauro Ballicchia ${ }^{1,2}$, , David K. Ferry ${ }^{3}\left(\mathcal{D}\right.$, Mihail Nedjalkov $^{2}$ and Josef Weinbub ${ }^{4, *(1)}$ \\ 1 Department of Information Engineering, Università Politecnica delle Marche, 60131 Ancona, Italy; \\ m.ballicchia@gmail.com \\ 2 Institute for Microelectronics, TU Wien, 1040 Wien, Austria; mihail.nedialkov@tuwien.ac.at \\ 3 School of Electrical, Computer, and Energy Engineering, Arizona State University, Tempe, AZ 85287-5706, \\ USA; ferry@asu.edu \\ 4 Christian Doppler Laboratory for High Performance TCAD, Institute for Microelectronics, TU Wien, \\ 1040 Wien, Austria \\ * Correspondence: josef.weinbub@tuwien.ac.at
}

Received: 23 January 2019; Accepted: 27 March 2019; Published: 30 March 2019

Featured Application: Modeling and simulation of quantum entanglement in physical systems.

\begin{abstract}
Quantum information and quantum communication are both strongly based on concepts of quantum superposition and entanglement. Entanglement allows distinct bodies, that share a common origin or that have interacted in the past, to continue to be described by the same wave function until evolution is coherent. So, there is an equivalence between coherence and entanglement. In this paper, we show the relation between quantum coherence and quantum interference and the negative parts of the Wigner quasi-distribution, using the Wigner signed-particle formulation. A simple physical problem consisting of electrons in a nanowire interacting with the potential of a repulsive dopant placed in the center of it creates a quasi two-slit electron system that separates the wave function into two entangled branches. The analysis of the Wigner quasi-distribution of this problem establishes that its negative part is principally concentrated in the region after the dopant between the two entangled branches, maintaining the coherence between them. Moreover, quantum interference is shown in this region both in the positive and in the negative part of the Wigner function and is produced by the superposition of Wigner functions evaluated at points of the momentum space that are symmetric with respect to the initial momentum of the injected electrons.
\end{abstract}

Keywords: coherence; entanglement; Wigner function; quasi-distribution; non-diagonal Wigner states; signed-particles; nanowire

\section{Quantum Information and The Wigner Function}

When we extend the classical idea of informatics, or even the idea of physically-based informatics to the quantum world, we uncover two basic threads: (1) quantum computing, and (2) quantum information as a description of quantum communications. The idea of using quantum mechanics to provide more computing power than classical computers seems to have begun around 1981-2 [1], but Deutsch put the ideas on a more firm basis a few years later [2]. Deutsch reformulated the Turing argument [3] with an assertion that every finite realizable physical system could be simulated in a perfect manner by a universal model computing machine of finite size, and even by a quantum mechanical version of this universal machine. The fact that the quantum computer can be far more efficient than a classical version relies upon the concept of entanglement, or quantum superposition [4]. Information in such a quantum computer is naturally quantum information, but we tend to use this 
term more for the transmission of information in a manner in which it cannot be intercepted. Naturally, the ideas of transmitting bits using quantum-based encryption grew along with the ideas of quantum computing, so the two together become quantum information processing $[5,6]$.

Important to each of these applications of quantum mechanics is the use of entanglement. When two bodies interact in a quantum manner they become entangled, which implies that they are now described by a single wave function and are no longer two distinct bodies. Even after they have ceased to interact and have moved some distance away, they remain entangled until some decoherence process acts upon them [7]. However, in experiments, one must face the fact that entanglement is difficult to measure. There is no physical variable whose eigenvalue yields the entanglement. Hence, people have worked for years to devise measures of entanglement [8]. Most of these, however, do not provide a clear visualization of the entanglement. But, it has been demonstrated that the Wigner function does provide a clear visualization of entanglement [7]. And, experimentalists have devised methods of reconstructing the Wigner function from output data of the experiments, particularly in optics (for a review on recent advances see [9]). One such approach involves the study of entanglement in the Jaynes-Cummings model [10]. This model studies the interaction between a cavity mode, treated as a harmonic oscillator, and a two-level atom. When the field in the cavity is in a coherent state, the atom is prepared in an excited state. It is found that the atomic and field states become rapidly entangled, and then subsequently disentangle at one-half the so-called revival time [11]. Thus, the maximum entanglement occurs when the atom Rabi oscillations are at maximum amplitude. It was subsequently demonstrated that the Wigner function can be written as a shifted expectation value of the optical parity operator [12]. Other optical experiments have shown that it is possible to measure directly a significant fraction of the Wigner function, when a spontaneous parametric down conversion of a photon produces a pair of entangled photons [13]. In some implementations of superconducting qubits, the entanglement can be used to follow the evolution of the Wigner function of the reduced density matrix of the qubits [14]. Indeed, it is now clear that the Wigner function can be used to exhibit directly the non-classical behavior through its non-Gaussian and negative excursions [15].

\subsection{The Wigner Function}

The remarkable similarities between the classical mechanics and the Wigner formulation of quantum mechanics offers a very convenient, and necessary, way to analyze coherence, entanglement, tunneling, and other characteristic effects of the quantum evolution. Indeed, both theories use phase space, defined by the position $x$ and momentum $p$, the physical observables are associated with the same functions $A(x, p)$ and importantly, the averaged physical quantities $\langle A\rangle$ are obtained by the same integrals

$$
\langle A\rangle=\int d x d p A(x, p) F(x, p),
$$

where $F$ is either the classical distribution function $f \geq 0$, or the Wigner function $f_{w}$. This establishes a unique correspondence between these functions and associates the name "quasi-distribution" to $f_{w}$. The basic difference is that the latter is still a real function, but allows negative values. This already suggests that these values are manifest of the quantum behavior and provide the kernel of the quantum information. The development of negative values of the Wigner function over phase space dimensions larger than $h$ are quantum features and the main focus of this work. As discussed above, these negative features can be associated with a rigorous quantification theory [16], developed in analogy with the corresponding theory of entanglement.

\subsection{Entanglement in the Wigner Function}

Coherence and entanglement are based on the superposition principle, however, they represent conceptually different ideas. The former reflects the wave nature of states to interfere: States with a fixed phase relationship superimpose to represent another state of the physical system. Thus, coherence can be represented in single quantum systems and it is always with respect to a particular basis. In turn, 
entanglement implicates the (tensor product of) states of two correlated systems in such a way, that a destruction of one of the states in the first system results in a change of the states in the second system.

Notably, the two concepts are quantitatively equivalent, that is, any nonzero amount of coherence in a system can be converted into an equal amount of entanglement between that system and another initially incoherent one [17]. This means that the two concepts which describe very different physical notions have a common mathematical foundation and thus can encrypt and carry the same information. The resources theory of coherence, which has been developed in terms of Hilbert space operator mechanics, designates a basis $\left|a_{i}\right\rangle$ and identifies a set of states $\mathcal{I}$ with a label incoherent. These concepts can be reformulated in phase space terms, moreover the entire theory can be formulated in the phase space. There is a variety of measures of coherence in complex systems, based on the correlations between the subsystems $[18,19]$.

A principal postulate in the development of a phase space quantum mechanics is the establishment of the correspondence between operators and functions. The problem is that different ordering of the position and momentum operators gives rise to different operators corresponding to one and the same observable, which is a function of the phase space coordinates. There are different rules for ordering the operators such as all momentum operators to the left or to the right, or a fully symmetric ordering. The Wigner theory is developed around the latter with the help of the Weyl map $A(x, p) \leftrightarrow \mathcal{W}(\hat{A})$ :

$$
\mathcal{W}(\hat{A})=\int \frac{d s d q}{h} \operatorname{Tr}\left(\hat{A} e^{\frac{i}{\hbar}(s \hat{x}+q \hat{p})}\right) e^{-\frac{i}{\hbar}(s x+q p)}
$$

The map establishes a one-to one correspondence between operators and functions, or more precisely an isomorphism from the algebra of operators $\hat{A}(\hat{x} \hat{p})$ with a product and a commutator [,] to the algebra of phase space functions $A(x, p)$ with a non-commutative star $(*)$-product $A * B=\mathcal{W}(\hat{A}$ - $\hat{B})$ and a Moyal bracket $i \hbar[A, B]_{\mathrm{M}}=\mathcal{W}([\hat{A}, \hat{B}])$. Recently, the algebraic notions used to derive the quantifying theory of coherence have been reformulated in terms of the Wigner theory [20].

In particular the nondiagonal eigenvector Wigner function $f_{i j}(x, p)$ is introduced in correspondence to the eigenvectors $\left|a_{i}\right\rangle$ of the operator $\hat{A}$ of a given physical observable:

$$
f_{i j}(x, p)=\frac{1}{h} \mathcal{W}\left(\left|a_{i}\right\rangle\left\langle a_{j}\right|\right) \leftrightarrow\left|a_{i}\right\rangle\left\langle a_{j}\right|
$$

The Weyl map allows the transfer of the concepts of the information theory of coherence to the Wigner phase space picture. We may then define coherence in terms of a fixed basis $\left|a_{i}\right\rangle$ in which one has to use (2) for the set of eigenvector Wigner functions $f_{i j}$ to define the incoherent states as

$$
\hat{\sigma}=\sum_{i} P_{i}\left|a_{i}\right\rangle\left\langle a_{i}\right| \leftrightarrow f_{w, \sigma}=\sum_{i} P_{i} f_{i i}
$$

where $P_{i}$ are probabilities. The set of such states are denoted by $\mathcal{I}$. Any other state which cannot be presented as in Equation (3) in this way is a coherent state [21]. Then the measure for coherence in phase space is $C\left(f_{w}\right)=\min \mathcal{D}\left(f_{w}, f_{w, \sigma}\right)$, where $f_{w, \sigma} \in \mathcal{I}$ is introduced from the measure for distance $\mathcal{D}[16,17]$. The latter can be based on the von Neumann entropy or trace distance [16], which can be equivalently defined in both operator and phase spaces. We conclude that incoherent states involve only diagonal elements $f_{i i}$ : From Equation (2) it follows that coherence is directly related to the existence of nondiagonal elements $f_{i j}$ in the state representation of $f_{w}$.

The above formal mathematical analysis developed in [20] can be directly linked to the negativity of the Wigner function and actually has a transparent physical background in terms of positive and negative particles. Indeed, if we choose $\hat{A}$ to be the momentum operator $\hat{A}=\hat{p}$ with a basis $\mathrm{e}^{i p j x / \hbar}$, the corresponding Wigner basis becomes

$$
f_{i j}(x, p)=e^{i x\left(p_{i}-p_{j}\right) / \hbar} \delta\left(\left(p_{i}+p_{j}\right) / 2 \pm p\right),
$$


which, due to the exponential prefactor, has the ability to form interference patterns and, in particular, to exhibit negative values. In contrast, for diagonal Wigner basis states, this prefactor becomes unity and (4) reduces to $\delta\left(p_{i} \pm p\right)$. Thus, according to Equation (3), incoherent states $f_{w, \sigma}$ have non-negative values. The associated physical picture is of classical particles with a distribution function $f(p)=$ $\sum_{i} P_{i} \delta\left(p_{i} \pm p\right)$. Here each particle "carries" a weight given by the probability $P_{i}$. In this way the Wigner picture reveals that the concept for incoherent states is deeply rooted in classical mechanics. In particular, the evolution of an initial particle distribution, subject to the action of a quadratic potential, is entirely classical: The Wigner potential reduces to a force, which governs particles over classical Newtonian trajectories. We can extend this picture to coherent Wigner states. The latter contain off-diagonal basis states, thus according to Equation (4) the weight of such particles should have complex values. Furthermore, we know that the Wigner function is a real quantity, thus after the cancellation of all imaginary values in the corresponding decomposition of the chosen coherent state, only positive and negative values remain. In this way, we come to two important conclusions:

- Quantum coherence is characterized by negative values of the Wigner function;

- In a virtual particle picture, coherent states are distinguished from classical states by the existence of particles with a negative weight.

These considerations are independent from the complexity of the system: The existence of subsystems is accounted for by a simple increase of the involved phase space coordinates.

An alternative approach uses the negativity of the Wigner function to define a parameter which measures the non-classicality of a given state based on the distance from a family of coherent states [22]. The parameter is given by the doubled volume of the integrated (in the whole phase space) negative part of the Wigner function. This definition can be regarded as a projection of the function onto the set of the real numbers between zero and unity. Here, we show that other ways of projection can provide useful information about the quantum evolution.

In the next section, we describe how entanglement can be observed in a simple physical problem, that of electron two-slit propagation around an impurity potential in a quantum nanowire (waveguide). The simulation experiment is very similar to the one already used in [23] to analyze coherence effects by comparing classical and quantum density or current density. In contrast, here, we reconstruct the Wigner function in the phase space in order to investigate how the negative excursions, not existing in the classical case, bear information about the involved quantum effects. We show that Wigner-quantum transport can be represented by the evolution of positive and negative particles: The so-called signed-particles. We then turn to the actual simulation and results in the following section.

\section{A Simple Example Problem and Methods}

\subsection{A Quasi-Two-Slit Electron Problem}

The aim of this paper is to illustrate how information on entanglement and coherence can be observed by means of the Wigner function of a physical system. The physical system that we consider consists of a portion of a nanowire with a repulsive dopant in the center of it, see Figure 1. The dopant potential creates a barrier in the center of the nanowire that causes the wave to break into two separate parts passing on either side of the barrier, much like two slits. Electrons that flow along the waveguide, interacting with the repulsive dopant, go through one slit or the other, generating a "Y-branch" path.

The lateral boundaries of the experimental setup are considered to be absorbing in order to avoid reflections of the two electron branches from the lateral sides of the nanowire. Minimum uncertainty non-interacting wave packets are injected from the bottom of the nanowire with a temporal spacing of $1 \mathrm{fs}$. The only source of quantum effects is the presence of the dopant potential that, having a spatial dependence of $1 / r$, presents derivatives of all orders.

The simulation set-up is very similar to that already used in [23]: The extent of the simulation domain is of $20 \times 30 \mathrm{~nm}^{2}$, with the dopant centered at $\left(x_{d}, y_{d}\right)=(10 \mathrm{~nm}, 15 \mathrm{~nm})$. The Wigner pure 
states are injected from $\left(x_{0}, y_{0}\right)=(10 \mathrm{~nm}, 0 \mathrm{~nm})$ in the $+y$ direction with an initial kinetic energy of $0.141 \mathrm{eV}$. The standard deviation of the minimum uncertainty wave packets is $3 \mathrm{~nm}$ in both spatial directions. The electron evolution has been simulated for $400 \mathrm{fs}$; this time is sufficient for the system to reach steady state. At the end of the simulation the Wigner function is analyzed, specifically to determine those regions of phase space where the quasi-distribution becomes negative.

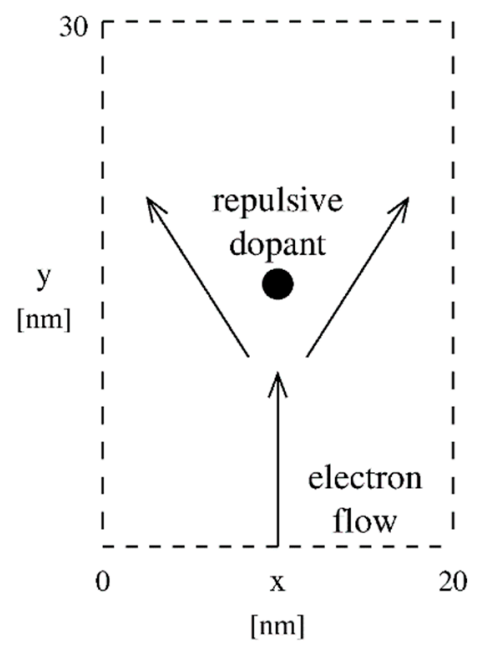

Figure 1. The two-slit electron problem: simulation setup.

\subsection{Signed-Particles}

Historically, the basic similarities between the concepts and notions of classical mechanics and quantum mechanics in phase space motivated the development of particle methods for solving the Wigner equation almost three decades ago [24]. Different particle approaches have been developed since then [25-27], which focus mainly on the electrostatic problem in a scalar potential gauge. In particular, the concept of signed-particles, which has matured for 15 years [28], is based on the application of stochastic approaches to solving integral equations by converting them to different integral forms of the Wigner equation. The concept is not a single, unique particle model, but comprises a set of particle attributes which can be combined and modified to develop suitable algorithms, specific for the physical aspects of the particular problem. Some of these attributes are, however, fundamental and give rise to a heuristic picture of quantum mechanics in terms of particles [29]. Basic attributes are particle sign, particle generation, and particle annihilation. In a signed-particle approach, point-like particles are enabled with classical features, such as drift over Newtonian (field-less) trajectories but carry the quantum information by their positive or negative sign. The mean value of a generic physical quantity $A$, represented by a phase space function, is evaluated by the $\sum_{n} \operatorname{sign}(n) A_{n}$ for all particles $n$ in a desired region, where $\operatorname{sign}(n)$ is the sign of the $n$-th particle and $A_{n}$ the corresponding value of the physical quantity. During the evolution, each particle generates couples of one positive and one negative particle, according to rules dictated by the Wigner potential, and propagate in space by distinct but fixed momentum (no acceleration). Particles with opposite sign which meet in the phase space annihilate each-other since they have a common probabilistic future but opposite contribution to the physical averages. Proof of concept simulations, showing that Newton's second law can be reproduced by generation/annihilation of un-accelerated signed-particles [29], have been conducted, and also for two- and three-dimensional problems [30]. This allows to prove that annihilation is able to reproduce classical ballistic behavior in the quantum case, where the applied electric field shows constant or linear behavior. Also, there aren't abrupt variations of the electric potential, like the one introduced by the dopant, that (exceeding quadratic dependence) generate quantum behavior. 


\section{Results}

The Wigner function for a two-dimensional scenario, as the one illustrated in Section 2.1, is a function of four variables, $f_{w}\left(x, y, k_{x}, k_{\mathrm{y}}\right)$ and thus needs a five-dimensional space to be visualized. However, useful information can be retrieved from the analysis of the projection of the function onto a two-dimensional space. From a mathematical point of view there are numerous ways to establish such a projection. Two of the variables, called variables of interest, remain active, while the remaining three must be restrained according to some criteria, usually introduced by physical considerations. For example, the value of the Wigner function and of one position and one momentum component can be fixed, or restricted in some limits, or integration on the latter within selected intervals can be performed.

As a first experiment we consider a separation of the negative and the positive part of the Wigner function: $f_{w}^{-}=f_{w} \theta\left(-f_{w}\right)$ and $f_{w}^{+}=f_{w} \theta\left(f_{w}\right)$. Then both quantities are integrated in the whole momentum space to obtain the spatial distribution of both the negative and the positive parts:

$$
f_{w_{x y}}^{-}(x, y)=\iint f_{w}^{-}\left(x, y, k_{x} k_{y}\right) d k_{x} d k_{y} ; f_{w_{x y}}^{+}(x, y)=\iint f_{w}^{+}\left(x, y, k_{x} k_{y}\right) d k_{x} d k_{y}
$$

We consider as a reference frame the classical (Boltzmann) distribution, characterized by: $f_{B_{x y}}^{-}(x, y)=0$ and $f_{B_{x y}}^{+}(x, y)=n(x, y)$ where $n$ is the classical density. Figure $2 \mathrm{a}, \mathrm{b}$ show respectively $f_{w_{x y}}^{-}$and $f_{w_{x y}}^{+}$. The quantum density, represented by the sum $f_{w_{x y}}^{+}+f_{w_{x y}}^{-}$is shown in Figure 3a. These quantities reflect the following evolution process. In the region below the dopant, denoted by the green $0.15 \mathrm{eV}$ isoline, the electron flux splits into two entangled parts. These maintain the coherence in the region after the dopant, as no dissipation processes are involved. This results in the interference fringes well visible in the upper parts after the dopant of both figures. Remarkably, $f_{w_{x y}}^{-}$exhibits firmly negative values in a wide region around the dopant. However, their pattern does not reflect the symmetry of the potential and thus they can't be associated to the nonlocality of the quantum action. The most pronounced negative values are placed after the dopant, in the region of evolution of the two entangled parts. Now, one can connect this negative region with the fact that, quantum mechanically, the scattering process requires a "collision duration" [31]. Quantum mechanically, the wave must first build up a correlation with the impurity, and this is then broken up when the collision is completed. The fact that the entanglement exists beyond the impurity is recognition that the event takes a non-zero amount of time to complete. This is true for the impurities as well as for phonons [32]. A similar push of the effect of the impurity potential to the anterior region can be caused by nonlinear screening due to dopants in the contacts [33], although no such screening is used here. In contrast, such behavior is entirely missing in Figure 3b, where the classical density $n$ is reported: The corresponding region is entirely empty! This shows that the negative excursions of the Wigner function provide a direct indicator for interference effects.

It is interesting to see if such analysis can be carried out on other types of projections or is specific to the presented one, where we intentionally separated the negative values of the Wigner function. As a second experiment we consider the Wigner function for two specific points in the momentum space that are $\left(k_{\mathrm{x} 1} k_{\mathrm{y} 1}\right)=\left(-0.26 \mathrm{~nm}^{-1}, 0.89 \mathrm{~nm}^{-1}\right)$ and $\left(k_{\mathrm{x} 2} k_{\mathrm{y} 2}\right)=\left(0.26 \mathrm{~nm}^{-1}, 0.89 \mathrm{~nm}^{-1}\right)$. The choice of these two points is related to the fact that they are near to the mean momentum along the $y$-direction $\left(0.84 \mathrm{~nm}^{-1}\right)$ and symmetrically placed around the initial momentum in the $x$-direction. Classical particles with such momentum coordinates exit near the upper corners of the simulation domain, thus entering well in the region of interest above the dopant, where we expect entanglement and interference effects in the quantum evolution. This can be seen in Figures $4 b, 5 b$ and $6 b$ presenting the classical distributions $f_{B}\left(x, y, k_{x 1}, k_{y 1}\right), f_{B}\left(x, y, k_{x 2}, k_{y 2}\right)$, and their sum, respectively. The choice of two specific symmetric points with respect to the initial momentum does not limit the analysis, since the evolution of the system is symmetric with respect to the initial momentum, and the choice of all the couples of symmetric points will give a full recovery of the Wigner function. For convenience the viewpoint in Figures 4-6 is now rotated, so that the exit (formerly upper) part of the simulation domain 
is now the closest one. The corresponding quantum function $f_{w}\left(x, y, k_{x 1}, k_{y 1}\right)$ shown in Figure $4 \mathrm{a}$ reveals a well-pronounced negative part, which begins around the dopant and is extended until the boundary. The same holds for the function $f_{w}\left(x, y, k_{x 2}, k_{y 2}\right)$, Figure $5 a$. We note that in both cases the negative parts are "internally" placed in the region of correlations between the left and right parts. In Figure 6a we report the distribution obtained by the sum $f_{w}\left(x, y, k_{x 1}, k_{y 1}\right)+f_{w}\left(x, y, k_{x 2}, k_{y 2}\right)$. The quantum distribution presents a negative part in front of the dopant between the two branches and a kind of "interference" behavior appearing due to the summation of the two components in that region. None of this can be seen in the corresponding classical evolution where the distribution between the two branches is zero.

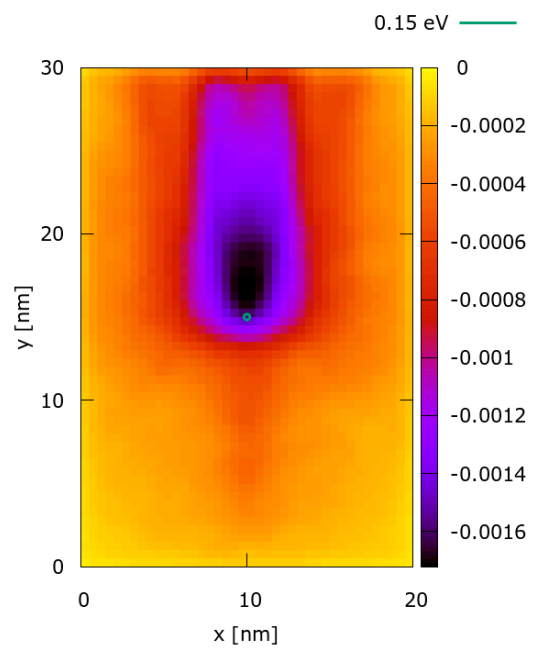

(a)

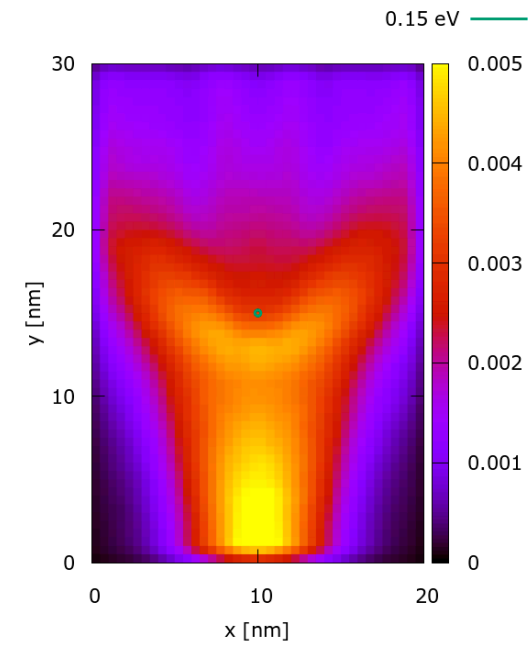

(b)

Figure 2. Spatial distribution of (a) the negative part $f_{w_{x y}}^{-}(x, y)$ and (b) the positive part $f_{w_{x y}}^{+}(x, y)$.

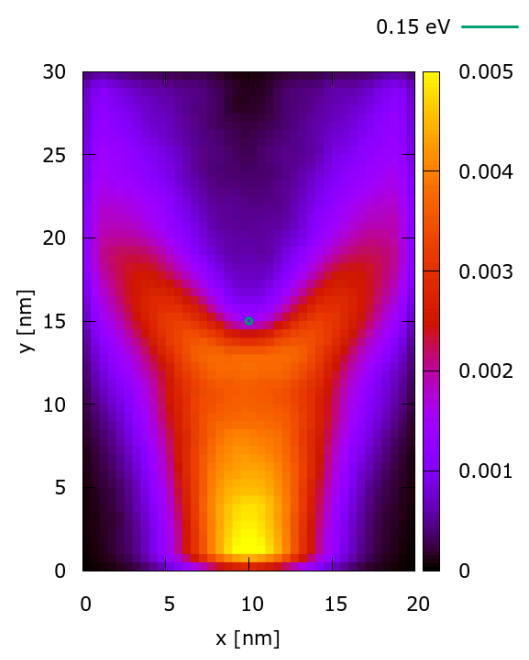

(a)

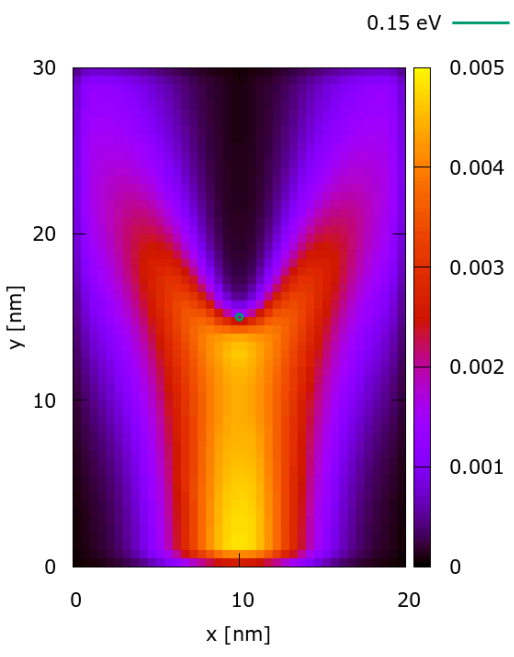

(b)

Figure 3. Comparison between quantum and classical distribution: (a) quantum distribution $n(x, y)=$ $f_{w_{x y}}^{+}(x, y)+f_{w_{x y}}^{-}(x, y),(\mathbf{b})$ classical distribution $n(x, y)=f_{w_{x y}}^{+}(x, y)$. 


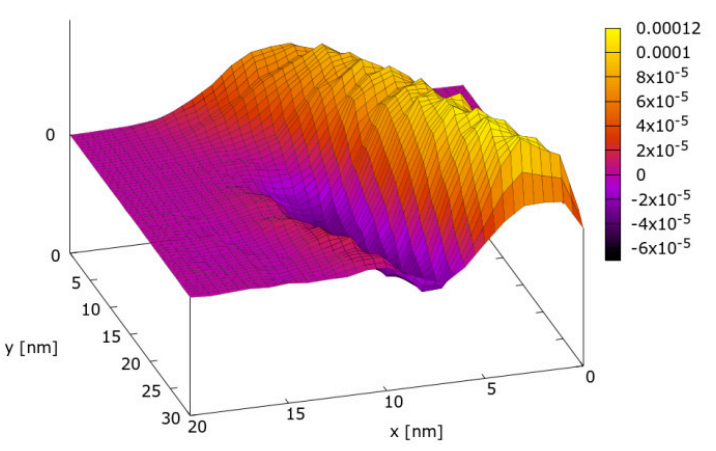

(a)

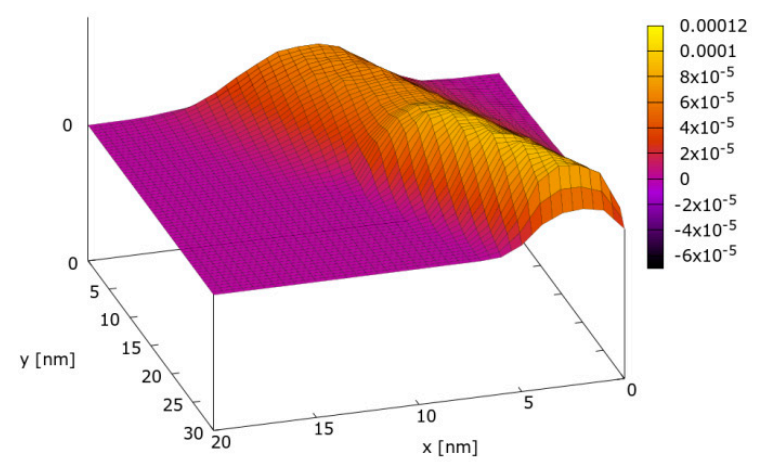

(b)

Figure 4. Comparison between quantum and classical phase-space distribution in the point $\left(k_{\mathrm{x} 1}\right.$, $\left.k_{\mathrm{y} 1}\right)=\left(-0.26 \mathrm{~nm}^{-1}, 0.89 \mathrm{~nm}^{-1}\right)$ : (a) quantum Wigner distribution, (b) corresponding classical Boltzmann distribution.

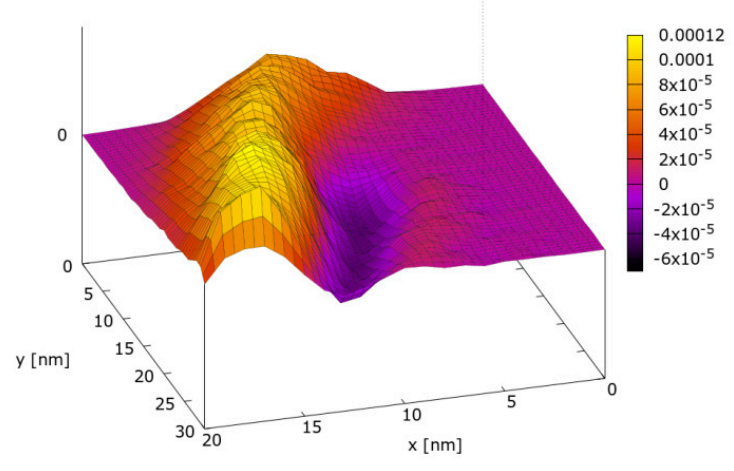

(a)

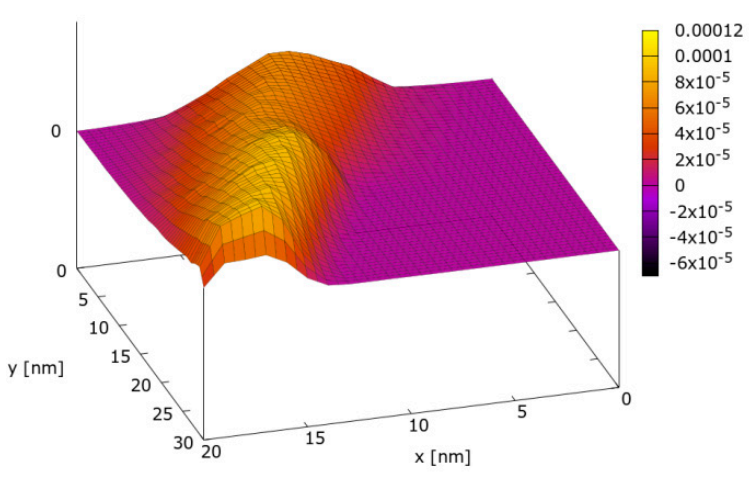

(b)

Figure 5. Comparison between quantum and classical phase-space distribution in the point $\left(k_{\mathrm{x} 2}, k_{\mathrm{y} 2}\right)=$ $\left(0.26 \mathrm{~nm}^{-1}, 0.89 \mathrm{~nm}^{-1}\right)$ : (a) quantum Wigner distribution, (b) classical Boltzmann distribution.

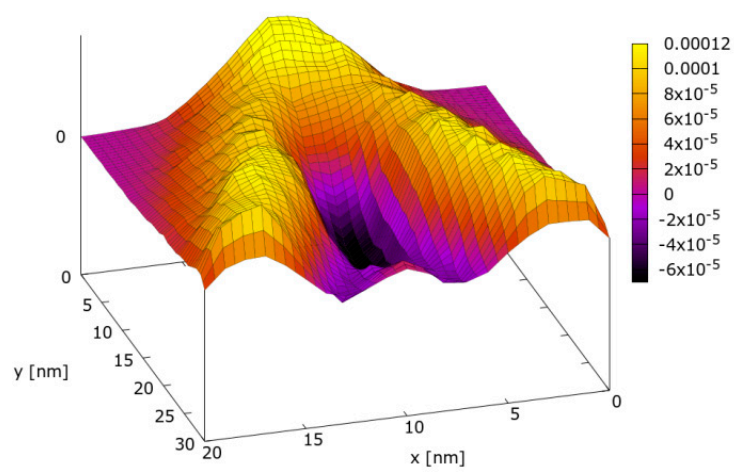

(a)

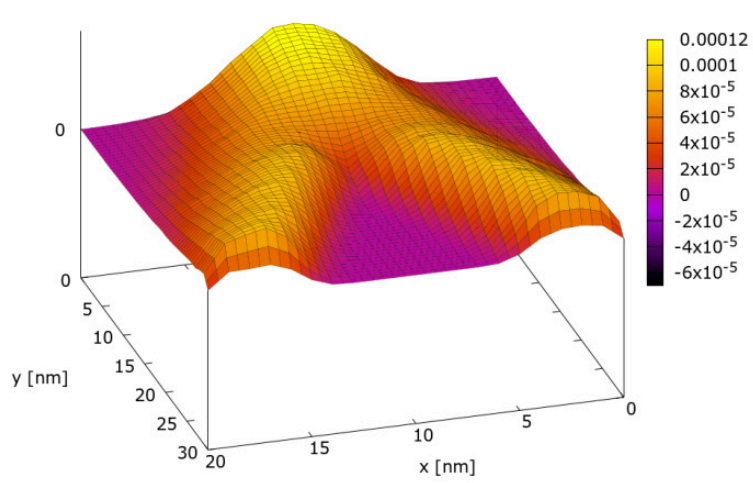

(b)

Figure 6. Comparison between quantum and classical sum of the phase-space distributions in the points $\left(k_{\mathrm{x} 1}, k_{\mathrm{y} 1}\right)=\left(-0.26 \mathrm{~nm}^{-1}, 0.89 \mathrm{~nm}^{-1}\right)$ and $\left(k_{\mathrm{x} 2}, k_{\mathrm{y} 2}\right)=\left(0.26 \mathrm{~nm}^{-1}, 0.89 \mathrm{~nm}^{-1}\right)$ : (a) quantum Wigner distribution, (b) classical Boltzmann distribution.

In this way, the information available in the first experiment can be directly retrieved from the second kind of projection, consistently providing a correlation between "negativity" and entanglement/interference. 


\section{Discussion and Conclusions}

The paper illustrates, by exploiting the definition of Wigner non-diagonal basis, the relation between the coherence and the negative part of the Wigner function. In particular, in the signed-particle formulation, quantum coherent states are distinguished from classical ones by the fact that the negative particles bring negative values. This shows that a Wigner signed-particle approach is a convenient way to analyze coherence and entanglement as it allows one to directly reconstruct the Wigner quasi-distribution. In order to show the validity of these considerations a simple physical problem of the electron evolution in a nanowire with a repulsive dopant placed in the center was considered. As observed, the active region of interaction around the dopant is characterized by negative values of the Wigner function, which can be associated to quantum nonlocality. However, the Wigner function values in the region after the dopant, characterized by the coherent evolution of the two entangled electron beams are considerably negative. This manifests the close link between coherent evolution and negative excursion of the Wigner function. In order to better show the link between coherence and negative excursion we have considered points in the momentum space, which are symmetric with respect to the initial momentum and are scattered by the dopant in two separated branches in the classical case. This enables to highlight that the corresponding quantum Wigner quasi-distribution presents a negative excursion in the inner part of these two branches and the superposition of these two negative excursions creates a pattern of interference between the two branches.

Author Contributions: Conceptualization, D.K.F. and J.W.; methodology, M.B. and M.N.; software, M.B.; validation, M.B., M.N., D.K.F., and J.W.; formal analysis, M.B., M.N., D.K.F., and J.W.; investigation, M.B., M.N., D.K.F., and J.W.; resources, M.N. and J.W.; data curation, M.B.; writing-original draft preparation, M.B.; writing-review and editing, M.N., D.K.F. and J.W.; visualization, M.B.; supervision, M.N., D.K.F. and J.W.; project administration, J.W.; funding acquisition, M.N. and J.W.

Funding: The financial support by the Austrian Science Fund (FWF): FWF-P29406-N30, the Austrian Federal Ministry for Digital and Economic Affairs, and the National Foundation for Research, Technology and Development is gratefully acknowledged.

Acknowledgments: The computational results presented have been achieved using the Vienna Scientific Cluster (VSC).

Conflicts of Interest: The authors declare no conflict of interest.

\section{References}

1. Benioff, P.A. Quantum mechanical Hamiltonian models of discrete processes that erase their own histories: Application to Turing Machines. Int. J. Theor. Phys. 1982, 21, 177-201. [CrossRef]

2. Deutsch, D. Quantum theory, the Church-Turing principle, and the universal quantum computer. Proc. Roy. Soc. London A 1985, 400, 97-117. [CrossRef]

3. Turing, A. On computable numbers, with an application to the Entscheidungs problem. London Math. Soc. Ser. II 1937, 42, 230-265. [CrossRef]

4. Schrödinger, E. Die gegenwärtige Situation in der Quantenmechanik. Naturwiss 1935, 23, 807-812, 823-828, 844-849.

5. Calderbank, A.R.; Shor, P.W. Good quantum error-correcting codes exist. Phys. Rev. A 1996, 54, $1098-1105$. [CrossRef]

6. Gea-Banacloche, J.; Kish, L.B. Comparison of energy requirements for classical and quantum information processing. Fluc. Noise Lett. 2003, 3, C3-C7. [CrossRef]

7. Ferry, D.K.; Nedjalkov, M. The Wigner Function in Science and Technology; IOP Publishing: Bristol, UK, 2018; Chapter 8.

8. Horodecki, R.; Horodecki, P.; Horodecki, M.; Horodecki, K. Quantum entanglement. Rev. Mod. Phys. 2009, 81, 865-942. [CrossRef]

9. Weinbub, J.; Ferry, D.K. Recent advances in Wigner function approaches. Appl. Phys. Rev. 2018, 5, 041104. [CrossRef]

10. Jaynes, E.T.; Cummings, F.W. Comparison of quantum and semiclassical radiation theories with application to the beam maser. Proc. IEEE 1963, 51, 89-109. [CrossRef] 
11. Gea-Banaloche, J. Collapse and revival of the state vector in the Jaynes-Cummings model: An example of state preparation by a quantum apparatus. Phys. Rev. Lett. 1990, 65, 3385-3388. [CrossRef]

12. Birrittella, R.; Cheng, K.; Gerry, C.C. Photon-number parity oscillations in the resonant Jaynes-Cummings model. Optics Commun. 2015, 354, 286-290. [CrossRef]

13. Douce, T.; Eckstein, A.; Walborn, S.P.; Khoury, A.K.; Ducci, S.; Keller, A.; Coudreau, T.; Milman, P. Direct measurement of the biphoton Wigner function through two-photon interference. Sci. Rep. 2013, 3, 3530. [CrossRef]

14. Reboiro, M.; Civitarese, O.; Tielas, D. Use of discrete Wigner functions in the study of decoherence of a system of superconducting flux-qubits. Phys. Scripta 2015, 90, 074028. [CrossRef]

15. Zhang, H.-L.; Yuan, H.-C.; Hu, L.-Y.; Xu, X.-X. Synthesis of Hermite excited squeezed vacuum states from two separate single-mode squeezed vacuum states. Opt. Commun. 2015, 356, 223-229. [CrossRef]

16. Baumgratz, T.; Cramer, M.; Plenio, M.B. Quantifying coherence. Phys. Rev. Let. 2014, 113, 140401. [CrossRef] [PubMed]

17. Streltsov, A.; Singh, U.; Dhar, H.S.; Bera, M.N.; Adesso, G. Measuring quantum coherence with entanglement. Phys. Rev. Lett. 2015, 115, 020403. [CrossRef] [PubMed]

18. Svozilík, J.; Vallés, A.; Peřina, J., Jr.; Torres, J.P. Revealing Hidden Coherence in Partially Coherent Light. Phys. Rev. Lett. 2015, 115, 220501. [CrossRef]

19. Kalaga, J.K.; Leoński, W.; Peřina, J., Jr. Einstein-Podolsky-Rosen steering and coherence in the family of entangled three-qubits states. Phys. Rev. A 2018, 97, 042110. [CrossRef]

20. Ellinghaus, P.; Weinbub, J.; Nedjalkov, M.; Selberherr, S. Analysis of lense-governed Wigner signed particle quantum dynamics. Phys. Stat. Sol. RRL 2017, 11, 1700102. [CrossRef]

21. Vitanov, P.; Nedjalkov, M.; Jacoboni, C.; Rossi, F.; Abramo, A. Unified Monte Carlo approach to the Boltzmann and Wigner equations. In Advances in Parallel Algorithms; Dimov, I., Tonev, O., Eds.; IOS Press: Amsterdam, Netherlands, 1994; pp. 154-196.

22. Kenfack, A.; Życzkowski, K. Negativity of the Wigner function as an indicator of non-classicality. J. Opt. B 2004, 6, 396-404. [CrossRef]

23. Ballicchia, M.; Weinbub, J.; Nedjalkov, M. Electron evolution around a repulsive dopant in a quantum wire: coherence effects. Nanoscale 2018, 10, 23037-23049. [CrossRef] [PubMed]

24. Jacoboni, C. Theory of Electron Transport in Semiconductors; Springer: Berlin/Heidelberg, Germany, 2010.

25. Querlioz, D.; Dollfus, P. The Wigner Monte Carlo Method for Nanoelectronic Devices; ISTE-Wiley: London, UK; Hoboken, NJ, USA, 2010.

26. Nedjalkov, M.; Querlioz, D.; Dollfus, P.; Kosina, H. Wigner Function Approach. In Nano-Electronic Devices, Semiclassical and Quantum Transport Modeling; Vasileska, D., Goodnick, S., Eds.; Springer: New York, NY, USA, 2011; pp. 289-358.

27. Nedjalkov, M.; Kosina, H.; Selberherr, S.; Ringhofer, C.; Ferry, D.K. Unified particle approach to Wigner-Boltzmann transport in small semiconductor devices. Phys. Rev. B 2004, 70, 115319. [CrossRef]

28. Ellinghaus, P. Two-Dimensional Wigner Monte Carlo Simulation for Time-Resolved Quantum Transport with Scattering. Doctoral Dissertation, TU Wien, Vienna, Austria, 2016. Available online: http:/ /www.iue. tuwien.ac.at/phd/ellinghaus / (accessed on 28 March 2019).

29. Nedjalkov, M.; Schwaha, P.; Selberherr, S.; Sellier, J.M.; Vasileska, D. Wigner quasi-particle attributes - An asymptotic perspective. Appl. Phys. Lett. 2013, 102, 163113. [CrossRef]

30. Weinbub, J.; Ballicchia, M.; Nedjalkov, M. Electron interference in a double-dopant potential structure. Phys. Stat. Sol. RRL 2018, 12, 1800111. [CrossRef]

31. Ferry, D.K. An Introduction to Quantum Transport in Semiconductors; Pan Stanford Publishing: Singapore, 2018.

32. Bordone, P.; Vasileska, D.; Ferry, D.K. Collision-duration time for optical-phonon emission in semiconductors. Phys. Rev. B 1996, 53, 3846. [CrossRef]

33. Towie, E.A.; Watling, J.R.; Barker, J.R. Remotely screened electron-impurity scattering model for nanoscale MOSFETS. Semicond. Sci. Technol. 2011, 26, 055008. [CrossRef]

(C) 2019 by the authors. Licensee MDPI, Basel, Switzerland. This article is an open access article distributed under the terms and conditions of the Creative Commons Attribution (CC BY) license (http://creativecommons.org/licenses/by/4.0/). 